\title{
PESQUISAS EM RECURSOS DE ALTA TECNOLOGIA PARA COMUNICAÇÃO E TRANSTORNO DO ESPECTRO AUTISTA
}

\author{
RESEARCH ON HIGH TECHNOLOGY RESOURCES FOR COMMUNICATION AND \\ AUTISM SPECTRUM DISORDER
}

\author{
INVESTIGACIONES EN RECURSOS DE ALTA TECNOLOGÍA PARA \\ COMUNICACIÓN Y TRANSTORNO DEL ESPECTRO AUTISTA
}

Carolina Rizzotto Schirmer ${ }^{1}$

\begin{abstract}
RESUMO
Cerca de um terço dos indivíduos diagnosticados com Transtorno do Espectro Autista (TEA) não conseguem desenvolver a oralidade. Os benefícios da Comunicação Alternativa (CA) para pessoas com TEA são bem reconhecidos e podem ser usados para ampliar a fala limitada ou atuar como o método de comunicação principal (ou seja, alternativo). Os dispositivos de geração de fala referem-se a uma variedade de soluções de alta tecnologia, incluindo dispositivos de comunicação eletrônica dedicados (comunicadores/vocalizadores), processadores de texto com voz sintetizada e dispositivos móveis multifuncionais (por exemplo:iPad, iPode tabletscom sistema Android) carregados com aplicativos de CA, e que são opções viáveis para indivíduos com autismo. O objetivo deste artigo é verificar os estudos que investigam a alta tecnologia para comunicação e TEA, publicados no Augmentative and Alternative Communication Journal, que é o periódico oficial da Sociedade Internacional de Comunicação Aumentativa e Alternativa (Isaac), no período de 2011 a 2018 . Esta é uma área nova de conhecimento que está em rápido crescimento e com poucos estudos no Brasil. Os resultados dos 15 artigos analisados indicam que pessoas com TEA se beneficiam com o uso da alta tecnologia. Pesquisas futuras em criação de recursos de comunicação, intervenção com usuários e descrição dos repertórios verbais são recomendadas.
\end{abstract}

PALAVRAS-CHAVE: Comunicação Alternativa. Transtorno do Espectro Autista. Alta Tecnologia. Revisão Sistemática.

\section{ABSTRACT}

About one-third of individuals diagnosed with Autism Spectrum Disorder (ASD) can not develop orality. The benefits of Augmentative and Alternative Communication (AAC) for individuals with ASD are well recognized and can be used to extend limited speech or act as the primary (ie alternative) communication method. Speech generation devices refer to a variety of high-tech solutions, including dedicated electronic communication devices (communicators / vocalizers), speech synthesizers and multifunctional mobile devices (eg iPad, iPod, and tablets with Android system) loaded with AC applications, and are a viable option for individuals with autism. The objective of this article is to verify the studies that investigate high technology for communication and ASD, published in the Augmentative and Alternative Communication Journal, which is the official journal of the International Society of Increasing and Alternative Communication (ISAAC), from 2011 to 2018. This is a new area of knowledge that is rapidly growing and with few studies in Brazil. The results of the 15 articles analyzed indicate that people with ASD benefit from the use of high technology. Future research on the creation of communication resources, intervention with users and description of verbal repertoires is recommended.

\footnotetext{
${ }^{1}$ Doutora em Educação - Universidade do Estado do Rio de Janeiro (UERJ) - Rio de Janeiro, RJ - Brasil. Professora - Universidade do Estado do Rio de Janeiro (UERJ) - Rio de Janeiro, RJ - Brasil. E-mail: ead.carolina@gmail.com.

Submetido em: 28/05/2019 - Aceito em: 16/10/2019
}

(C) ETD- Educação Temática Digital Campinas, SP $\quad$ v.22 n.1 $\quad$ p.68-85 jan./mar.2020 
KEYWORDS: Augmentative and Alternative Communication. Autism Spectrum Disorder. High Tech. Systematic Review.

\section{RESUMEN}

Cerca de un tercio de los individuos diagnosticados con trastorno del espectro autista (TEA) no pueden desarrollar la oralidad. Los beneficios de la Comunicación Alternativa (CA) para personas con TEA son bien reconocidos y pueden ser usados para ampliar el habla limitada o actuar alternativamente como el método de comunicación principal. Entre los dispositivos de generación de habla encontramos una variedad de soluciones de alta tecnología, incluyendo dispositivos de comunicación electrónica dedicados (comunicadores/vocalizadores), procesadores de texto con voz sintetizada y dispositivos móviles multifunción (por ejemplo, iPad, iPod y tablets con sistema Android) con aplicaciones de CA; todos ellos son opciones viables para los individuos con autismo. El objetivo de este artículo es analizar os estudios que aplicaron a alta tecnología para comunicación y TEA, publicados en el Augmentative and Alternative Communication Journal, que es el periódico oficial de la Sociedad Internacional de Comunicación Aumentativa y Alternativa (ISAAC), durante el período de 2011 a 2018. Esta es una nueva área de conocimiento que está en rápido crecimiento y con pocos estudios en Brasil. Los resultados de los 15 artículos analizados indican que las personas con TEA se benefician con el uso de la alta tecnología. Las investigaciones futuras en la creación de recursos de comunicación, la intervención con los usuarios y la descripción de los repertorios verbales se recomiendan.

PALAVRAS-CLAVE: Comunicación Alternativa. Trastorno del Espectro Autista. Alta Tecnología. Revisión Sistemática.

\section{INTRODUÇÃO}

De acordo com a Associação Americana de Psiquiatria (APA, 2013), o Transtorno do Espectro Autista (TEA) é caracterizado por déficits na comunicação social e comportamentos restritos e repetitivos. Segundo os Centros de Controle e Prevenção de Doença dos Estados Unidos (CDC, 2014), aproximadamente uma em cada 68 crianças tem diagnóstico de TEA. Cerca de um terço destes indivíduos não conseguem desenvolver a oralidade, ou seja, têmdeficiências severas na comunicação e não são capazes de se comunicar apenas pela fala. Assim, apoiar as habilidades de comunicação de crianças, jovens e adultos com autismo ${ }^{2}$ é um componente essencial de intervenção com essa população. Os benefícios da Comunicação Alternativa ${ }^{3}$ (CA) para indivíduos com TEA são bem reconhecidos e podem ser usados para ampliar a fala articulada limitada (não funcional) ou atuar como o método de comunicação principal (ou seja, alternativo), aumentando sua participação nas atividades cotidianas em casa, na escola e em outros contextos na comunidade (KAGOHARA et al., 2013; LIGHT; MCNAUGHTON, 2012, 2013).

\footnotetext{
${ }^{2}$ Neste estudo, os termos autismo e autistaserão utilizados para se referir a pessoa com Trasntrono do Espectro Autista.

${ }^{3}$ Comunicação Alternativa é uma área da prática clínica ou educacional que visa complementar, ampliar ou substituir a fala ou a escrita convencional por meio de estratégias, técnicas, recursos e serviços (BEUKELMAN; MIRENDA, 2013).
} 
Os Sistemas de Comunicação Altenativa (SCA) são divididos em duas categorias. A primeira são os sistemas sem auxílio externo - como gestos, sinais manuais e vocalizações -e, a segunda, os com auxílio externo organizados em recursos de baixa tecnologia, média e alta tecnologia que podem utilizar apoios visuais (como simbolos pictográficos), considerados importantes para ajudar a comunicação e a interação em pessoas com TEA (BOESH et al., 2013; GANZ et al., 2012; SCHLOSSER; WENDT, 2008). Os recursos de baixa tecnologia são aqueles que costumam ter menor custo e são fáceis de ser confeccionados como: pranchas, livros e cartões de comunicação com simbologia gráfica, fotografias e letras. Os de média tecnologia também são chamados de Dispositivos de Geração de Fala (DGF) dedicados ou especialmente desenvolvidos para a CA. São eles os recursos de comunicação com saída de voz que produzem fala digitalizada ou sintetizada, conhecidos como comunicadores/vocalizadores que não envolvem uso de software/aplicativo. A alta tecnologia são os DGFs que empregam tecnologia eletrônica ou de computador complexa. Referem-se a uma variedade de soluções, incluindo dispositivos de comunicação eletrônica dedicados (comunicadores/vocalizadores), processadores de texto com voz sintetizada e dispositivos móveis multifuncionais (por exemplo: celulares, $\mathrm{iPad}^{\mathrm{TM}}$, $\mathrm{iPod}^{\mathrm{TM}}$, tablets com sistema Android ${ }^{\mathrm{TM}}$ ) carregados com softwares/aplicativos de CA (KAGOHARA et al., 2013; LIGHT; MCNAUGHTON, 2013). Todos os SCA são uma opção viável para indivíduos com TEA. Porém o uso de dispositivos móveis multifuncionais utilizados como recursos de comunicação e educação estão se expandindo rapidamente na comunidade (GANZ et al., 2017; KAGOHARA et al., 2013; LIGHT; MCNAUGHTON; CARON,2019), principalmente quando falamos em autismo. Ao realizarmos uma simples busca ${ }^{4}$ no aplicativo Play Store, no sistema operacional Android ${ }^{\mathrm{TM}}$, utilizando a palavra-chave "autista", podemos localizar, pelo menos, duzentos aplicativos (apps) com objetivos variados (informativos de saúde, jogos, educação etc.). Ao analisarmos esses apps e organizarmos pelas categorias propostas pelo Play Store, a que apresenta o maior número de apps é a de Ensino, com 73, seguida das categorias de Educação, com 44, e de Comunicação, com 16.

O uso de alta tecnologia na CA aumentou significativamente nos últimos anos, principalmente em virtude da acessibilidade e portabilidade das tecnologias móveis, tais como telefones celulares e tablets com tela sensível ao toque (LIGHT; MCNAUGHTON, 2013; STILL et al., 2014). Um dos aspectos que torna esses recursos mais acessíveis, especificamente tecnologias móveis, se dá pelo fato de o custo dos apps de CA ser inferior aos tradicionais DGFs dedicados (LIGHT; MCNAUGHTON, 2013) que, na nossa realidade brasileira, possuem um valor mais alto, já que se trata de uma tecnologia importada e que,

\footnotetext{
${ }^{4}$ Pesquisa realizada no período de setembro de 2018 a novembro de 2018, pela graduanda Juliana Tavares, para seu Trabalho de Conclusão de Curso na Graduação de Pedagogia da Universidade do Estado do Rio de Janeiro.
} 
apesar de ser direito da pessoa com deficiência e TEA, não costuma ser concedido pelo governo. Still et al. (2014) referem que os aplicativos de CA utilizados em tecnologia móvel, geralmente, são considerados simples de programar e também são menos estigmatizantes do que uma prancha de comunicação ou cartões com imagens. Com isso, é fundamental que essas novas formas de CA sejam exploradas, pesquisadas e avaliadas.

É fato que, muitas crianças e jovens com TEA e/ou outras deficiências são incapazes de satisfazer suas necessidades diárias de comunicação apenas com a fala articulada. Assim, a inclusão social e educacional para esses indivíduos é um desafio e, por isso, os SCA representam um importante apoio às interações comunicativas e sociais do usuário ou potencialmente usuário de CA com seus pares com desenvolvimento típico, principalmente no caso de alunos autistas incluídos no sistema regular de ensino. Porém a CA, seja ela de baixa, média ou alta tecnologia, por enquanto não está acessível a todos que precisam. Existem ainda muitos mitos em relação ao seu uso (como a ideia equivocada de que o uso da CA vai impedir o desenvolvimento da fala), e faltam profissionais habilitados para implementar métodos, técnicas, estratégias e recursos de CA. Mas, por outro lado, temos a facilidade de baixar aplicativos de CA que, às vezes, estão disponíveis gratuitamente em nossos telefones celulares e tablets, todavia, o que fazer com esses apps? Como programálos? Como ensinar e inserir seu uso nas atividades cotidianas?

Vários estudos apontam que o recurso pelo recurso não é suficiente para que uma pessoa aprenda e utilize um SCA com eficácia. Light e McNaughton (2013) ressaltam o perigo de que a excitação sobre estes novos recursos de tecnologia móvel associados a apps de CA possa resultar em um foco equivocado na tecnologia, no recurso, favorecendo a negligência do que deveria ser o foco central - as pessoas com necessidades complexas de comunicação que requerem CA. Os autores afirmam que para se usufruir do potencial que a tecnologia oferece, profissionais da educação e da saúde devem garantir que a intervenção da CA seja conduzida não pelos dispositivos, mas sim pelas necessidades de comunicação deste indivíduo. Tendo em vista que a CA é uma área em desenvolvimento no Brasil e que estudos nacionais sobre o uso da alta tecnologia ainda são poucos, buscamos, por meio deste artigo, responder à seguinte questão: o que as pesquisas publicadas no Augmentative and Alternative Communication Journal (AAC), importante periódico da área, revelam sobre o uso de alta tecnologia para pessoas com TEA? 


\section{METODOLOGIA}

A revisão sistemática da literatura realizada neste artigo utilizou como base de dados as publicações do Augmentative and Alternative Communication Journal (AAC), no período de 2011 a 2018. O AACé o periódico oficial da Sociedade Internacional de Comunicação Aumentativa e Alternativa ${ }^{5}$ (International Society for Augmentative and Alternative Communication -Isaac), no qual são publicados trimestralmente artigos científicos relacionados com o campo da $\mathrm{CA}$, que relatam pesquisas sobre avaliação, tratamento, reabilitação e educação de pessoas que usam ou têm potencial para usar CA, bem como promovem discussões sobre teoria, tecnologia e desenvolvimento de SCA relevantes para a área. Nesse periódico, os artigos são submetidos a uma revisão cega por pares na língua inglesa. Para o levantamento das publicações, foram utilizados os descritores Augmentative and Alternative Communication, High Technology e Autism.Os critérios de inclusão adotados foram: artigos originais, ano de publicação de 2011 a 2018, que tratassem do tema da alta tecnologia na CA para pessoas com TEA. Compreendendo a alta tecnologia como os DGF: vocalizadores (comunicadores), computadores, tecnologia móvel (celulares e tablets) com uso de apps para a CA. As revisões descritivas e os estudos de pesquisa meta-analítica publicados também foram incluídos.

Inicialmente, foram localizados 97 artigos no sistema da editora Taylor \& Francis Online ${ }^{6}$, responsável pela publicação do periódico. Após a leitura minuciosa dos títulos, das palavras-chave e dos resumos foram selecionados 15 trabalhos que possuíam os descritores utilizados, estabelecendo-se também como critério de seleção aqueles com aderência à temática estudada. As razões pelas quais 81 artigos foram excluídos são: ausência de participantes com TEA; revisões descritivas e meta-analíticas que não tivessem o foco no TEA e alta tecnologia e estudos que não tivessem utilizado a alta tecnologia. Por exemplo, no estudo desenvolvido por Boesh et al. (2013) que comparou o Picture Exchange Communication System - PECS (BONDY; FROST, 1994) com um DGF de média tecnologia.

A seguir, no Quadro 1, são descritos os 15 artigos incluídos que serão analisados.

\footnotetext{
${ }^{5}$ Disponível em: https://www.isaac-online.org/english/home/

${ }^{6}$ Disponível em:

https://www.tandfonline.com/action/journallnformation?show=aimsScope\&journalCode=iaac20\#.VcjssvlVhBc
}

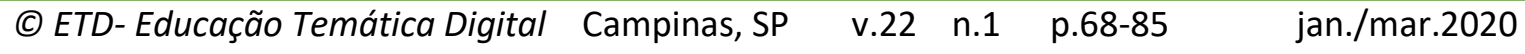


QUADRO 1.Artigos incluídos

\begin{tabular}{|c|c|c|}
\hline Autores & Título & Ano \\
\hline $\begin{array}{l}\text { Trottier; } \\
\text { Mirenda }\end{array}$ & $\begin{array}{l}\text { Effects of Peer-Mediated Instruction to Teach Use of Speech- } \\
\text { Generating Devices to Students with Autism in Social Game } \\
\text { Routines }\end{array}$ & 2011 \\
\hline Flores et al. & $\begin{array}{l}\text { A Comparison of Communication Using the Apple iPad and a } \\
\text { Picture-based System }\end{array}$ & 2012 \\
\hline Schlosser et al. & $\begin{array}{l}\text { Implementing Directives that Involve Prepositions with Children } \\
\text { with Autism: A Comparison of Spoken Cues with Two Types } \\
\text { of Augmented Input }\end{array}$ & 2013 \\
\hline Meer et al. & $\begin{array}{l}\text { Teaching Multi-Step Requesting and Social Communication to } \\
\text { Two Children with Autism Spectrum Disorders with Three AAC } \\
\text { Options }\end{array}$ & 2013 \\
\hline $\begin{array}{l}\text { Gillespie-Smith; } \\
\text { Fletcher-Watson }\end{array}$ & $\begin{array}{l}\text { Designing AAC Systems for Children with Autism: Evidence from } \\
\text { Eye Tracking Research }\end{array}$ & 2014 \\
\hline Schlosser; Koul & Individuals with Autism Spectrum Disorders: A Scoping Review & 2015 \\
\hline Ganz & $\begin{array}{l}\text { AAC Interventions for Individuals with AutismSpectrum Disorders: } \\
\text { State of the Science and Future Research Directions }\end{array}$ & 2015 \\
\hline Stephenson & $\begin{array}{l}\text { Using the Choiceboard Creator }{ }^{T M} \text { app on an iPadC to teach choice } \\
\text { making to a student with severe disabilities }\end{array}$ & 2016 \\
\hline Ganz et al. & $\begin{array}{l}\text { High-technology augmentative and alternative communication } \\
\text { for individuals with intellectual and developmental disabilities } \\
\text { and complex communication needs: a meta-analysis }\end{array}$ & 2017 \\
\hline Alzrayer; Banda; Koul & $\begin{array}{l}\text { Teaching children with autism spectrum disorder and other } \\
\text { developmental disabilities to perform multistep requesting using } \\
\text { an iPad }\end{array}$ & 2017 \\
\hline Genc-Tosun; Kurt & $\begin{array}{l}\text { Teaching multi-step requesting to children with autism spectrum } \\
\text { disorder using systematic instruction and a speech-generating } \\
\text { device }\end{array}$ & 2017 \\
\hline Ryan et al. & $\begin{array}{l}\text { Responsiveness of a parent-reported outcome measure to } \\
\text { evaluate AAC interventions for children and youth with complex } \\
\text { communication needs }\end{array}$ & 2018 \\
\hline $\begin{array}{l}\text { Donato; Spencer; } \\
\text { Arthur-Kelly }\end{array}$ & $\begin{array}{l}\text { A critical synthesis of barriers and facilitators to the use of } A A C \text { by } \\
\text { children with autism spectrum disorder and their } \\
\text { communication partners }\end{array}$ & 2018 \\
\hline Caron et al. & $\begin{array}{l}\text { Effects of dynamic text in an AAC app on sight word reading for } \\
\text { individuals with Autism spectrum disorder }\end{array}$ & 2018 \\
\hline Morin et al. & $\begin{array}{l}\text { A systematic quality review of high-tech AAC interventions as an } \\
\text { evidence-based practice }\end{array}$ & 2018 \\
\hline
\end{tabular}

Fonte: Dados da pesquisa.

Os seguintes procedimentos de análise de dados foram adotados: primeiramente, o autor leu na íntegra todos os artigos, em seguida, eles foram resumidos, com o objetivo de expor informações qualitativas referentes à alta tecnologia em CA e TEA. Foram destacados os objetivos, os participantes, o delineamento de pesquisa e os principais resultados relacionados ao foco deste artigo. 


\section{RESULTADOS}

Dos quinze artigos incluídos nessa revisão sistemática, seis eram de revisão e utilizaram diferentes métodos como: revisão de literatura (DONATO; SPENCER; ARTHURKELLY, 2018; GANZ, 2015; GILLESPIE-SMITH; FLETCHER-WATSON, 2014), revisão de escopo(SCHLOSSER; KOUL, 2015), meta-análise(GANZ et al.,2017) e revisão sistemática (MORIN et al., 2018). Oito eram de pesquisas experimentais (ALZRAYER; BANDA; KOUL,2017; CARON et al., 2018; FLORES et al., 2012; GENC-TOSUN; KURT,2017; SCHLOSSER et al., 2013; STEPHENSON, 2016; TROTTIER; KAMP; MIRENDA, 2011; MEER et al., 2013) e um era estudo de coorte (RYAN et al. (2018). Os resultados das análises qualitativas dos artigos estão descritos a seguir e organizados pelos anos de publicação de 2011 a 2018.

Trottier, Kamp e Mirenda (2011) investigaram os efeitos de uma intervenção mediada por pares, projetada para ensinar duas crianças com TEA a usar DGF para se engajarem em interações com os parceiros em um contexto social na escola. Seis colegas com desenvolvimento típico (três colegas de cada aluno com TEA) foram ensinados a apoiar o uso do DGF durante atividades de jogo. Foram utilizados dois modelos diferentes de DGF com símbolos PCS, ambos recomendados pela equipe de Educação Especial que os acompanhava. Os DGF foram programados com o vocabulário necessário à participação nos jogos, escolhidos de acordo com os interesses dos alunos com TEA. Um delineamento de linha de base múltipla foi utilizado para examinar a relação entre a instrução mediada por pares e um aumento nos atos comunicativos totais pelos dois alunos com TEA. As sessões instrucionais aconteceram em salas de aula da escola. Os resultados forneceram evidências de que os colegas adquiriram as habilidades necessárias para apoiar o uso do DGF pelos alunos com TEA. Também sugerem que a intervenção foi eficaz em aumentar o número total de atos comunicativos apropriados por estudantes com TEA. Além disso, os índices de validade social de todos os colegas foram positivos.

Flores et al. (2012) investigaram a funcionalidade do iPad $^{\mathrm{TM}}$ como um DGF, comparando seu uso a um SCA não eletrônico com símbolos gráficos PCS. Participaram cinco alunos do Ensino Fundamental, sendo que três deles possuíam diagnóstico de TEA. $O$ estudo experimental foi realizado em uma escola. A hora do lanche foi escolhida para a intervenção, por ser considerada um momento natural para comunicação incidental e de interesse dos alunos. A frequência dos comportamentos de comunicação foi comparada sob duas condições: um SCA com Intercâmbio de Figuras (IF) e um com iPad ${ }^{\text {TM }}$. Os resultados indicaram diferenças na frequência de comportamentos de comunicação comparando os dados nas condições do sistema com IF e no iPad ${ }^{\mathrm{TM}}$. Os comportamentos de comunicação aumentaram ao usar tablet ou permaneceram iguais ao usar cartões com símbolos. Com relação à preferência, que não foi um objetivo do estudo, alguns dados qualitativos sugerem que dois dos alunos preferiram o iPad ${ }^{\mathrm{TM}}$ ao IF. Os professores relataram, na pesquisa de

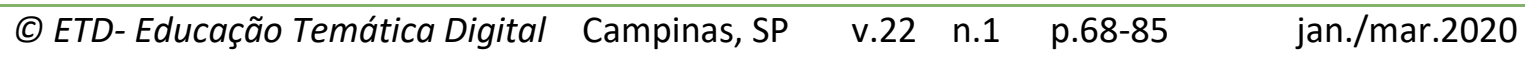


validade social, que optaram por usar um sistema como o iPad ${ }^{\mathrm{TM}}$, em vez do Intercâmbio de Figuras (IF), por causa de sua facilidade de implementação.

Schlosser et al. (2013) tiveram como objetivo comparar o input da fala com duas modalidades de input (fala + pista visual estática, fala + pista visual dinâmica), no que se refere à capacidade de as crianças com TEA seguirem ordens que envolviam preposições. Ou seja, nesse estudo, o foco era a linguagem receptiva. A pesquisa foi experimental, e envolveu nove participantes com TEA. Nela, foi utilizadoo iPad ${ }^{\mathrm{TM}}$ para a apresentação das pistas visuais dinâmicas. Os resultados sugerem que pessoas com TEA são mais capazes de seguirem ordens quandoestas são dadas por meios aumentados, ou seja, empregando modalidades visuais e auditivas, em oposição ao uso da modalidade auditiva sozinha. E que tanto as pistas de cenas estáticas (fotografias) quanto as de cenas dinâmicas (vídeos) foram mais eficazes do que as dicas faladas, mas não houve diferenças entre as pistas de cena estática e dinâmica.

Meer et al. (2013) desenvolveram um estudo com vários objetivos, entre eles, o principalfoi verificar se usuários de CA podem aprender habilidades mais complexas e socialmente orientadas de comunicação com diferentes SCA, e se eles demonstram preferência. Fizeram parte da pesquisa duas crianças com TEA, que já haviam participado de estudos anteriores, nos quais aprenderam a solicitar itens de interesse por meio dos seguintes SCA: IF, Sinais Manuais (SM) e DGF. A referida pesquisa foi experimental, com delineamento alternado, utilizando diferentes recursos de alta tecnologia com app de CA. Durante a linha de base, os participantes demonstraram baixos níveis de resposta comunicativa correta. Com a intervenção, ambos aprenderam as respostas-alvo (duas e três etapas, solicitando respostas, saudações, respondendo perguntas e respostas de etiqueta social, como por favor e obrigado), com diferentes níveis de proficiência em cada opção de comunicação. Ambos participantes alcançaram o IF, porém um demonstrou preferência pelo DGF e, o outro, por IF.

Gillespie-Smith e Fletcher-Watson (2014) realizaram uma revisão da literatura sobre Rastreamento Ocular (RO) em indivíduos com TEA, com o objetivo de extrair resultados que tivessem implicações no desenvolvimento de SCA, analisando 22 estudos publicados entre os anos de 2002 e 2014. As evidências do RO eos efeitos do conteúdo social ${ }^{7}$, do uso de imagens realistas, complexas, com texto eas implicações do uso destes na construção dos SCA foram analisados. As conclusões, porém, são limitadas pelo fato de que poucos estudos foram projetados para verificar as consequências deste conhecimento aos SCA. As pesquisas, em geral, incluem indivíduos jovens e adultos com TEA, que têm uma linguagem

${ }^{7}$ Conteúdo social refere-se à presença de pessoas ou rostos em símbolos e imagens usadas nos SCA. Estes regularmente incluem rostos e figuras humanas, para representar conceitos como sentimentos, ações e certos substantivos (GILLESPIE-SMITH; FLETCHER-WATSON, 2014). 
intacta e sem Deficiência Intelectual (DI). Por isso, ainda é desconhecido o comportamento relativo ao olhar das crianças com TEA e DI, que são um grupo mais propenso a precisar do uso da CA. Os autores relatam que os padrões de movimento ocular atípico são mais encontrados nos participantes com mais deficiências severas na comunicação. Os estudos sugerem uma relação estreita entre a fixação e a localização e atenção, enquanto a relação entre o olhar e a compreensão é muito menos clara. Tais pesquisas foram conduzidas principalmente em laboratórios controlados, sendo pouco conhecida a influência de um parceiro comunicativo ou ambiente de sala de aula no comportamento do olhar desses alunos. Estudos de RO sugerem que as pessoas com autismo podem ser menos capazes de modificar seus movimentos oculares em resposta à demanda de tarefas, em comparação com indivíduos com desenvolvimento neurotípico. Outro aspecto que eles salientam é o fato de que a maioria das pesquisas de RO é conduzida empregando novos estímulos, como fotografias mostrando pessoas desconhecidas,com configurações também desconhecidas, ao contrário dos símbolos utilizados nos SCA.

Schlosser e Koul (2015) realizaram uma revisão de escopo com o objetivo de mapear evidências de pesquisa feitas até o momento sobre a eficácia das intervenções, incluindo DGF de alta tecnologia para indivíduos com TEA. Os autores localizaram 48 estudos (47 desenhos experimentais de caso único e 1 ensaio clínico randomizado) envolvendo 187 indivíduos. Os resultados foram revisados, a partir da divisão em três grupos de estudos: (a) estudos que avaliaram a eficácia de um conjunto de procedimentos de intervenção envolvendo o volume de fala; (b) estudos comparando um conjunto de procedimentos de intervenção com outras modalidades de CA; e (c) estudos para comparar a presença com a ausência de saída da fala. Em relação ao primeiro grupo, 26 estudos avaliaram os efeitos dos DGFs como parte de umconjunto de procedimentos de intervenção, envolvendo um total de 116 participantes. Foram utilizados 13 modelos diferentes de DGFs, três tipos de tecnologias móveis com dois diferentes apps.Os autores consideraram que oito estudos forneceram evidência conclusivas de que houve aumento no volume de fala, seis foram classificados como preponderantes, sete foram classificados como sugestivos e cinco foram avaliados como inconclusivo. Em relação às comparações de uma condição que abrangia um resultado de fala com outras modalidades de CA foram localizados 17 estudos, com um total de 53 participantes.As pesquisas, de modo geral, realizaram uma comparação para examinar a eficácia de uma abordagem de CA envolvendo um DGF em relação à outra modalidade de CA. Quanto às modalidades de CA empregadas, a maioria dos estudos comparou os DGFs com o uso de qualquer IF ou a abordagem com o PECS, além do uso de SM como uma terceira condição de comparação. Foram utilizadosquatro modelos diferentes de DGFs dedicados, quatro tipos de tecnologia móvel e três apps. E, por último, os autores encontraram cinco estudos que compararam o uso de quatro modelos de DGF com Ausência de Fala de Saída. Participaram 18 crianças com autismo. O impacto do uso da saída da fala foi examinado em três áreas: ortografia de palavras, solicitação e produção da fala.

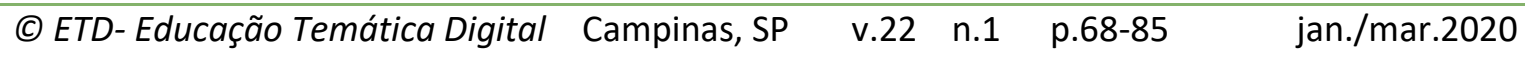


Os autores concluíram que esta revisão verificou o aumento das pesquisas na área. Eles referem que as investigações atuais de avaliação de preferência dos participantes em uma modalidade de CA em relação a outra são bem concebidas e consistentes. Os pesquisadores optaram pela estratégia de delineamento de pesquisa quase experimental em grupo ou estudo de caso único.

Ganz (2015) realizou uma revisão bibliográfica com três objetivos: (a) resumir e sintetizar as últimas décadas de pesquisa sobre o uso de CA por pessoas com TEA; (b) indicar as implicações dos estudos para as partes interessadas; e (c) delinear prioridades para que futuras pesquisas na área possam aperfeiçoar a intervenção e SCA. Ela relata que nas últimas décadas um grande corpo de pesquisa, principalmente com delineamento experimental de caso único, demonstrou a eficácia de CA para pessoas com TEA. Estudos foram publicados sobre várias modalidades de CA, incluindo SM, IF e DGF. Eles apontam que SCAs de baixa e alta tecnologia, investigados através de meta-análises e revisões sistemáticas, demonstraram a eficácia tanto no uso do IF quanto no uso dos DGFs, porém a autora ressalta que, especificamente, as tecnologias móveis são uma área emergente, e que precisam ser mais estudadas. Quanto aos delineamentos que reduzem as demandas de aprendizado e maximizam a atuação, a autora cita a tecnologia do RO. No que se refere ao contexto no qual a CA é implementada, os estudos têm investigado vários ambientes, incluindo os educacionais, com parceiros comunicativos variados, ressaltando que estes espaços podem ser tão eficazes quanto os estruturados. Além disso, um pequeno número de estudos indicou que a implementação de intervenções em CA por variados parceiros de comunicação, incluindo pais, colegas, professores, pode ser tão eficaz quanto à implementação por pesquisadores ou especialistas.

Stephenson (2016) descreve uma intervenção para ensinar o uso de um iPad ${ }^{\mathrm{TM}}$ comapps de CA, para que um aluno com TEA e DI pudesse fazer escolhas. O estudo com um delineamento de linha de base múltipla foi desenvolvido de forma colaborativa com os profissionais da escola. A intervenção foi projetada para se encaixar à rotina de classe especial, e seus resultados foram muito variáveis. Esta prolongou-se por quatro meses e foi concluídaem virtudedo final do ano letivo. Com o término da intervenção conduzida pelo professor, em sala de aula, o estudante fez escolhas válidas com 11 imagens, em três diferentes momentos. Os dados sugerem a preferência do uso do $\mathrm{iPad}^{\mathrm{TM}}$ pelo estudante.Embora a devida cautela deva ser usada, dados o comportamento e o elevado número de ensaios sem resposta na avaliação inicial, parece que a intervenção pode ter contribuído para uma melhoria geral nas habilidades de solicitar por meio de símbolos.

Ganz et al. (2017) tiveram como objetivo realizar uma meta-análise de pesquisas experimentais de caso único sobre o uso de alta tecnologia na $\mathrm{CA}$, por indivíduos com TEA e outras deficiências. Os autores localizaram 24 publicações e constataram que os recursos de 
alta tecnologia em CA foram eficazes. Todas as funções comunicativas analisadas tiveram efeito positivo com a intervenção. Infelizmente, a maioria dos autores avaliou expressão de desejos e necessidades, e poucos levaram em conta o impacto de comportamentos comunicativos sociais mais complexos. O estudo também verificou o impacto de se implementar a CA em contextos naturais versus didáticos-instrução, bem como do uso de CA por um pesquisador ou pais e cuidadores, mostrando que não houve diferença significativa entre eles.

Alzrayer, Banda e Koul (2017) realizaram um estudo com o objetivo de determinar a eficácia da instrução sistemática no ensino de habilidades para solicitar em várias etapas, usando um DGF associado a aplicativo de CA. Participaram deste estudo quatro crianças, sendo que três foram diagnosticadas com TEA e DI, com habilidades de comunicação funcional não verbais ou muito limitadas. O estudo experimental foi desenvolvido em salas de aula estruturadas para a educação especial em escolas primárias. Os resultados indicaram que todos os participantes adquiriram a capacidade de navegar e combinar símbolos para solicitar itens ou atividades preferenciais. Três deles também foram capazes de generalizar as habilidades conquistadas, mediante pedido de novos itens ou atividades preferenciais.

Genc-Tosun e Kurt (2017) examinaram a eficácia de utilizar um iPad ${ }^{\mathrm{TM}}$ como SCA em um programa de intervenção no ensino de várias etapas, para que crianças com TEA aprendam a habilidade de solicitar. O programa de intervenção incluiu ensino experimental discreto, atraso de tempo, orientação graduada e reforço. Participaram do estudo três alunos com TEA, com habilidades de comunicação funcional nãoverbal ou muito limitada.Todas as sessões foram realizadas em uma sala de pesquisa. A pesquisa experimental utilizou o delineamento com linha de base múltipla. Os resultados da pesquisa indicaram que o conjunto de procedimentos de intervenção usando um DGF com app de CA foi eficaz no ensino da habilidade de solicitar através de múltiplas etapas para todas as crianças. Os três participantes também mantiveram as habilidades adquiridas durante uma, duas, quatro e 12 semanas após as sessões de treinamento, que foram generalizadas por meio de diferentes ferramentas e pessoas. Os resultados desse estudo sugeriram que os participantes, com nenhuma história anterior quanto ao usode um DGF, aprenderam a solicitar através deste novo recurso e desenvolveram a habilidade para discriminar entre símbolos. Os dados de validade socialindicaram que os parceiros de comunicação tiveram opiniões positivas sobre o estudo em geral.

Ryan et al. (2018) desenvolveram um estudo de coorte com o objetivo de verificar o potencial da Escala de Impacto na Família da Tecnologia Assistiva para CA (Family Impact of Assistive Technology Scale for AAC -Fiats-AAC), visando detectar mudança em crianças e jovens com necessidades complexas de comunicação (TEA, paralisia cerebral, síndrome de 
Rett, síndrome de Down, entre outras), e suas famílias, após seis e 12 semanas em que os usuários receberam um DGF com símbolos gráficos. A maioria dos participantes (82\%) recebeu seu primeiro DGF no decorrer do estudo. Os DGFs entregues aos usuários envolveram uso de seis diferentes hardwares e sete diferentes softwares. Ao todo, 45 pais completaram o Fiats-AAC. Como resultados, verificaram que o estudo fornece suporte inicial para a capacidade de o Fiats-AAC detectar mudanças em crianças e jovens e suas famílias depois de receber e utilizar o novo recurso de comunicação. Os pais relataram que seus filhos incluíram os DGFs em suas interações cotidianas com familiares, parceiros de comunicação, e os clínicos indicaram que os participantes tiveram suas competências comunicativas aumentadas nas semanas após a recepção do novo SCA.

Donato, Spencer e Arthur-Kelly (2018) realizaram uma revisão da literatura com o propósito de sintetizar de forma crítica as barreiras e os facilitadores para o uso de SCA por crianças com TEA e seus parceiros de comunicação. A busca foi limitada ao período de 20042016. A análise dos dados dos 66 estudos incluídos foi qualitativa, através de metodologia de síntese crítica. Tais estudos compreenderam desenhos de estudo quaseexperimentais, não experimentais e qualitativos, e relataram a gama completa de SCA utilizada. Como resultados, os autores apontaram as seguintes barreiras: dificuldades no acesso a serviços de intervenção para seus filhos; quanto às políticas que restringem o acesso de crianças a serviços e/ou esquemas de financiamento quando atingem uma determinada idade; prestadores de serviços que não têm formação necessária para usar um SCA e prestam um aconselhamento inconsistente às famílias; e treinamento ou tempo necessários para criar ou programar SCA. No que concerne ao uso de alta tecnologia, são citados problemas com recursos que exigiam energia da bateria para operar, dificuldades para navegar no software, e que, nem sempre, estava disponível ou acessível para crianças dentro e entre diferentes contextos. Facilitadores descritos incluíram um aumento da intensidade da intervenção da CA e o apoio oferecido às famílias pelos prestadores de serviços que tinham experiência na CA. Entre os facilitadores relacionados à alta tecnologia de CA, está a seguinte situação: quando os SCAs estavam disponíveis para as crianças em todos os seus ambientes naturais, era fácil para elas e seus parceiros de comunicaçãofazerem uso deles; quando os dispositivos tinham uma variedade de funções, incluindo tecnologia de foto e vídeo, registro eletrônico do progresso das crianças e adaptação do vocabulário conforme as necessidades destas, reduziam o estigma.

Caron et al. (2018) desenvolveram um estudo que investigou os efeitos dos recursos de um software que possui texto dinâmico e saída de fala na seleção de um símbolo gráfico dentro de uma prancha em um app de CA. Participaram do estudo cinco crianças com TEA, que possuíam habilidades de comunicação funcional nãoverbais ou muito limitadas e que frequentavam classes especiais. Os estímulos usados nas fases do estudo foram baseados em um total de 15 palavras, 12 direcionadas para a aprendizagem de todos os participantes

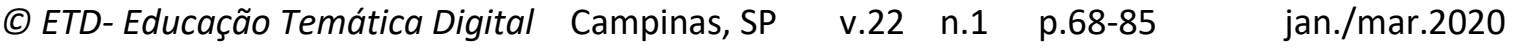


(escolhidas pelo foco de interesse) e três utilizadas pelo pesquisador para modelar os componentes operacionais das tarefas. Os recursos do softwareT2L foram introduzidos usando um DGF dedicado, que foi programado com 15 botões e com os mesmos símbolos gráficos usados nas tarefas de sondagem. Todas as sessões ocorreram em uma sala de aula. Foi desenvolvido um estudo experimental com delineamento de linha de base múltipla com sujeito único. Todos os participantes aprenderam a reconhecer visualmente as palavras. Além disso, generalizaram sua aprendizagem para uma prancha de CA somente com texto - demonstrando a transição de uma prancha de CA baseada em símbolos gráficos para uma prancha com palavras.

Morin et al. (2018) realizaram uma revisão sistemática com o objetivo de avaliar a qualidade das pesquisas experimentais de delineamento de caso único sobre o uso de CA de alta tecnologia, para ensinar habilidades de comunicação social para indivíduos com TEA ou DI. Além disso, informações sobre os seguintes padrões metodológicos são relatadas em todos os estudos incluídos: descrição do participante, descrição do local e materiais, descrição dos procedimentos. Os resultados das pesquisasmostraram que o uso de CA de alta tecnologia para ensinar habilidades de comunicação social a indivíduos com TEA ou DI, que apresentem necessidades complexas de comunicação, pode ser considerado uma prática baseada em evidências. A revisão de comparação de estudos não indicou que a CA de alta tecnologia é significativamente melhor do que a CA de baixa tecnologia.

\section{DISCUSSÃO E CONCLUSÃO}

Todos os estudos analisaram e buscaram coletar resultados, identificar lacunas e apontar caminhos para futuras pesquisas na área da alta tecnologia para CA e TEA, principalmente descrevendo intervenções e implicações para a avaliação e desenvolvimento desses sistemas de alta tecnologia. Diferentes modelos de DGFs dedicados foram citados (CARON et al., 2018; RYAN et al., 2018;TROTTIER; KAMP; MIRENDA, 2011), a tecnologia móvel mais utilizada foi o iPad $^{\text {TM }}$ (ALZRAYER; BANDA; KOUL, 2017; FLORES et al., 2012; GENC-TOSUN; KURT, 2017; RYAN et al., 2018; SCHLOSSER et al., 2013; STEPHENSON, 2016; MEER et al., 2013) e os apps descritos foram: Proloquo2Go ${ }^{\mathrm{TM}}$ (ALZRAYER; BANDA; KOUL, 2017; RYAN et al., 2018; MEER et al., 2013), Pick a Word ${ }^{\text {TM }}$ (FLORES et al., 2012),

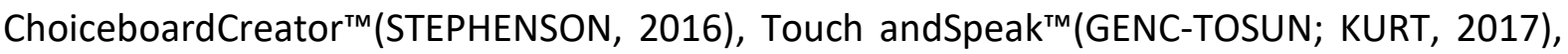
Minspeak $^{\mathrm{TM}}$, Unity ${ }^{\mathrm{TM}}$, LAMP Words for Life $^{\mathrm{TM}}$, TouchChat ${ }^{\mathrm{TM}}$, Communicator ${ }^{\mathrm{TM}}$, Dynavox Compass $^{\mathrm{TM}}$, WordPower ${ }^{\mathrm{TM}}$ e Predictable ${ }^{\mathrm{TM}}$ (RYAN et al., 2018), Pecs de Fase III (SCHLOSSER; KOUL, 2015), além do softwarepara alfabetização T2L (CARON et al., 2018).

Os estudos evidenciam a eficácia da intervenção com alta tecnologia de CA em pessoas com TEA, para ampliar a comunicação (ALZRAYER; BANDA; KOUL, 2017; FLORES et al., 2012; GANZ, 2015;GENC-TOSUN; KURT, 2017; GILLESPIE-SMITH; FLETCHER-WATSON, 2014; RYAN et al., 2018; SCHLOSSER; KOUL, 2015; STEPHENSON, 2016; TROTTIER; KAMP;

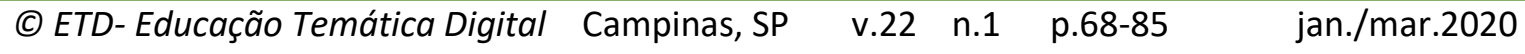


MIRENDA, 2011; MEER et al., 2013). Os mesmos resultados também foram encontrados nos estudos realizados por Kagohara et al.(2013), Kasari et al. (2014) e Stephenson e Limbrick (2015).

Os pesquisadores citam que DGFs de alta tecnologia possuem recursos ausentes na baixa tecnologia, que se mostram eficazes como o input aumentado (fala + pista visual estática, fala + pista visual dinâmica) (SCHLOSSER et al., 2013), a ampliação da imagem (símbolo gráfico ou fotografia) quando esta é selecionada e a discriminação visual dos símbolos, pois a cada seleção variam as posições na prancha (STEPHENSON, 2016). Porém é importante ressaltar que os estudos que compararam os SCAs de alta, média e baixa tecnologia não encontraram divergências significativas com relação à eficácia quando confrontados com os diferentes sistemas (FLORES et al., 2012; GANZ, 2015; MORIN et al., 2018; MEER et al., 2013).

Em relação à preferência, os dados sugerem que a alta tecnologia pode ser uma modalidade de escolha mais frequente entre indivíduos com TEA (FLORES et al., 2012; SCHLOSSER; KOUL, 2015; STEPHENSON, 2016; MEER et al., 2013). Outros pesquisadores compararam SCAs não eletrônicos e DGF em relação à preferência e à aquisição dos recursos, e seus estudos demonstraram que o tipo de sistema não afeta as taxas de aquisição, mas que os alunos podem mostrar preferências individuais por tipos específicos de SCA (ACHMADI et al., 2014; CANELLA- MALONE; DEBAR; SIGAFOOS, 2009).

O estudo de Donato, Spencer e Arthur-Kelly (2018) aponta que parceiros de comunicação demonstram que o interesse das crianças e a motivação para usarem SCA de alta tecnologia facilitariam a sua implementação. Ganz (2015) discute que a tecnologia móvel com software de CA tem vantagens sobre o uso de IF e DGF dedicados, que incluem menor custo, o que permite que as famílias tenham acesso mais fácil à $C A$, portabilidade (mais leves e fáceis de carregar), extensão de vocabulário, além de serem atraentes e comumente associados a atividades de reforço, sem falar na aceitabilidade de dispositivos móveis em toda a comunidade. Outra questão importante é o fato de o aplicativo ser rapidamente programado e fácil de ser utilizado, durante as atividades cotidianas, quando surge a necessidade de acréscimo de vocabulário, por exemplo. Além disso, a programação pode ocorrer diretamente no DGF, não necessitando usar o recurso do computador para depois transferir o conteúdo. Esses aplicativos permitem que pais e profissionais possam tirar fotos ou vídeos (usandouma câmera do celular, por exemplo) para capturar eventos ou experiências.A capacidade de programação no momento em que surge a necessidade de comunicar algo garante que parceiros de comunicação possam responder ao interesses do usuário acrescentando vocabulário relevante no decorrer das interações com ele, durante todo o dia (LIGHT; MCNAUGHTON; CARON, 2019). 
Outros aspectos importantes também são levantados, como a questão da introdução dos recursos em contextos estruturados ou naturais (escola), mostrando que nesses dois ambientes as intervenções são eficazes, como também serão se a implementação for realizada por um especialista ou parceiro de comunicação (GANZ, 2015; GANZ et al., 2017). Os estudos experimentais analisados neste artigo foram desenvolvidos em diferentes contextos (escolas especiais e regulares, laboratórios) e as intervenções realizadas também foram feitas, em alguns casos, por pesquisadores, professores ou colegas. O que parece ser mais evidente em todas as pesquisas é a necessidade de um programa sistematizado de intervenção que contemple os interesses do usuário de CA, assim como suas habilidades e potencialidades, pois, segundo Light e McNaughton (2013), a revolução tecnológica e seus desafios no campo da CA já são uma realidade. As autoras criticam o fato de que, às vezes, a intervenção em CA é interpretada apenas como um instrumento que fornece a tecnologia em CA, como se conceder um recurso, com treino limitado, fosse o suficiente para uma pessoa com necessidades complexas de comunicação ter sucesso na sua comunicação e interação social.

Após os estudos analisados neste artigo, podemos concluir que a alta tecnologia para CA em pessoas com TEA é eficaz, emergente e apropriada para o desenvolvimento das habilidades de comunicação e interação. E que o desenvolvimento de competência comunicativa de pessoas com TEA é impactado por inúmeros fatores, incluindo aqueles relacionados à pessoa, ao meio ambiente e aos parceiros de comunicação, bem comopelos diferentes SCAs (LIGHT; MCNAUGHTON, 2013).

É importante ressaltar que maispesquisas são necessárias para avaliar a alta tecnologia na CA, principalmente a tecnologia móvel associada a aplicativos de CA. É essencial que sejam produzidos estudos nacionais na área que contemplem nossa cultura e realidade, além dos recursos tecnológicos desenvolvidos em nosso país. As pesquisas devem ser organizadas com rigor metodológico, comparando os diferentes SCAs, a presença e a ausência de produção de fala. É fundamental, ainda, elaborar programas de intervenção que busquem criar habilidades comunicativas mais complexas.

\section{REFERÊNCIAS}

ACHMADI, Donna et al. Undergraduates' perceptions of three augmentative and alternative communication modes. Developmental Neurorehabilitation, v. 18, n. 1, p. 22-25, 2015.

ALZRAYER, Nouf M.; BANDA, Devender R.; KOUL, Rajinder. Teaching children with autism spectrum disorder and other developmental disabilities to perform multistep requesting using an iPad. Augmentative and Alternative Communication, v. 33, n. 2, p. 65-76, 2017.

AMERICAN PSYCHIATRIC ASSOCIATION - APA. Diagnostic and statistical manual of mental disorders (DSM-V). Washington, DC: American Psychiatric Association, 2013.

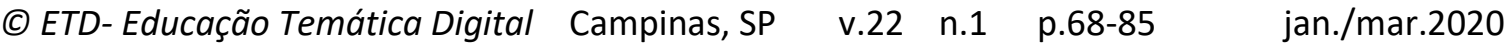


BEUKELMAN, David R.; MIRENDA, Pat. Augmentative and alternative communication: supporting children and adults with complex communication needs. 4th ed. Baltimore: Paul H. Brookes Publishing Co, 2013.

BOESCH, Miriam C. et al. Comparative efficacy of the picture exchange communication system (pecs) versus a speech-generating device: effects on social-communicative skills and speech development. Augmentative and Alternative Communication, v. 29, n. 3, p. 197209, 2013.

BONDY, Andy; FROST, Lori. PECS: the picture exchange communication system training manual. Cherry Hill, NY: Pyramid Educational Consultants, 1994.

CANELLA-MALONE, Helen; DEBAR, Ruth M.; SIGAFOOS, Jeff. An examination of preference for augmentative and alternative communication devices with two boys with significant intellectual disabilities. Augmentative and Alternative Communication, v. 25, n. 4, p. 262-273, 2009.

CARON, Jessica et al. Effects of dynamic text in an AAC app on sight word reading for individuals with autism spectrum disorder. Augmentative and Alternative Communication, v. 34 , n. 2, p. 143-154, 2018.

CENTERS FOR DISEASE CONTROL AND PREVENTION - CDC. Prevalence of autism spectrum disorder among children aged 8 years - Autism and Developmental Disabilities Monitoring Network. MMWR Surveillance Summary, United States, v. 63, p. 1-21, 2014.

DONATO, Cynthia; SPENCER, Elizabeth; ARTHUR-KELLY, Michael. A critical synthesis of barriers and facilitators to the use of AAC by children with autism spectrum disorder and their communication partners. Augmentative and Alternative Communication, v. 34, n. 3, p. 242-253, 2018.

FLORES, Margaret et al. A comparison of communication using the Apple iPad and a picturebased system. Augmentative and Alternative Communication, v. 28, n. 2, p. 74-84, 2012.

GANZ, Jennifer B. AAC interventions for individuals with autism spectrum disorders: state of the science and future research directions. Augmentative and Alternative Communication, v. 31, n. 3, p. 203-214, 2015.

GANZ, Jennifer B. et al. High-technology augmentative and alternative communication for individuals with intellectual and developmental disabilities and complex communication needs: a meta-analysis. Augmentative and Alternative Communication, v. 33, n. 4, p. 224238, 2017.

GANZ, Jennifer B. et al. A meta-analysis of single case research studies on aided augmentative and alternative communication systems with individuals with autism spectrum disorders. Journal Autism Dev Disord, v. 42, n. 1, p. 60-74, 2012. 
GENC-TOSUN, Derya; KURT, Onur. Teaching multi-step requesting to children with autism spectrum disorder using systematic instruction and a speech-generating device.

Augmentative and Alternative Communication, v. 33, n. 4, p. 213-223, 2017.

GILLESPIE-SMITH, Karri; FLETCHER-WATSON, Sue. Designing AAC systems for children with autism: evidence from eye tracking research. Augmentative and Alternative

Communication, v. 30, n. 2, p. 160-171, 2014.

KAGOHARA, Debora M. et al. Using iPodsand iPadsin teaching programs for individuals with developmental disabilities: a systematic review. Research in Developmental Disabilities, v. 34, p. 147-156, 2013.

KASARI, Connie et al. Communication interventions for minimally verbal children with autism: A sequential multiple assignment randomized trial. Journal of the American Academy of Child \& Adolescent Psychiatry, v. 53, p. 635-646, jun. 2014.

LIGHT, Janice; MCNAUGHTON, David. Putting People First: re-thinking the role of technology in augmentative and alternative communication intervention. Augmentative and Alternative Communication, v. 29, n. 4, p. 299-309, 2013.

LIGHT, Janice; MCNAUGHTON, David. The changing face of augmentative and alternative communication: past, present, and future challenges. Augmentative and Alternative Communication, v. 28, n. 4, p. 197-204, 2012.

LIGHT, Janice; MCNAUGHTON, David; CARON, Jessica. New and emerging AAC technology supports for children with complex communication needs and their communication partners: state of the science and future research directions. Augmentative and Alternative Communication, v. 35, n. 1, p. 26-41, 2019.

MEER, Larah et al. Teaching multi-step requesting and social communication to twochildren with autism spectrum disorders with three AAC options. Augmentative and Alternative Communication, v. 29, n. 3, p. 222-234, 2013.

MORIN, Kristi L. et al. A systematic quality review of high-tech AAC interventions as an evidence-based practice. Augmentative and Alternative Communication, v. 34, n. 2, p. 104117, 2018.

RYAN, Stephen E. et al. Responsiveness of a parent-reported outcome measure to evaluate AAC interventions for children and youth with complex communication needs.

Augmentative and Alternative Communication, v. 34, n. 4, p. 348-358, 2018.

SCHLOSSER, Ralph W.; KOUL, Rajinder K. Speech output technologies in interventions for individuals with autism spectrum disorders: a scoping review. Augmentative and

Alternative Communication, v. 31, n. 4, p. 285-309, 2015. 
SCHLOSSER, Ralph W.; WENDT, Oliver. Effects of augmentative and alternative communication intervention on speech production in children with autism: a systematic review. American Journal of Speech-Language Pathology, v. 17, p. 212-230, 2008.

SCHLOSSER, Ralph W. et al. Implementing Directives that involve prepositions with children with autism: a comparison of spoken cues with two types of augmented input.

Augmentative and Alternative Communication, v. 2, n. 2, p. 132-145, 2013.

STEPHENSON, Jennifer. Using the Choiceboard Creator ${ }^{\mathrm{TM}}$ app on an iPadC to teach choice making to a student with severe disabilities. Augmentative and Alternative

Communication, v. 32, n. 1, p. 49-57, 2016.

STEPHENSON, Jennifer; LIMBRICK, Lisa. A review of the use of touch-screen mobile devices by people with developmental disabilities. Journal of Autism and Developmental Disorders, v. 45, n. 12, p. 3777-3791, 2015.

STILL, Katharine et al. Facilitating requesting skills using high-tech augmentative and alternative communication devices with individuals with autism spectrum disorders: a systematic review. Research in Autism Spectrum Disorders, v. 8, p. 1184-1199, 2014.

TROTTIER, Nadine; KAMP, Lorraine; MIRENDA, PAT. Effects of peer-mediated instruction to teach use of speech-generating devices to students with autism in social game routines.

Augmentative and Alternative Communication, v. 27, n. 1, p. 26-39, 2011.

Revisão gramatical realizada por:

Vânia Coutinho Santiago.

E-mail: vaniacsantiago@hotmail.com. 